\title{
Cloud-MANET and its Role in Software-Defined Networking
}

\author{
Tanweer Alam \\ Department of Computer Science, Islamic University of Madinah, 170 Prince Naif Ibn Abdulaziz Road, Al Jamiah, Madinah, 42351 Saudi Arabia

ABSTRACT The telecommunication networking technologies are changing day by day with the advancement of the existing technologies. In the proposed $5 G$ networks, the role of Software-defined networking (SDN) is to provide programmable networking and decoupling the control and data plane. SDN comes from the requirement for providing the dynamic, high speed with low latency connectivity in real-time. This requirement introduces a new framework for creating programmable networking, decoupling forwarding and controlling planes, flexible real-time network, virtualizations in networking for managing the complexities in $5 \mathrm{G}$ heterogeneous networking. The emergence of virtualization has become the most useful technology for creating virtual machines remotely and apply security rules using programmable hardware to control centrally. In this article, the author discussed the cloud-based mobile ad hoc network (Cloud-MANET or sometimes called C-MANET) in the architecture of SDN for $5 \mathrm{G}$ heterogeneous networks. The main idea of creating the CMANET mobility model in the SDN framework is to enable programmable networking and accessibility for the moving devices in the range of MANET and cloud connectivity. In the framework of SDN, the C-MANET mobility model implementation is not an easy task, but it can be a very useful contribution to add this model in the framework of SDN so that it provides the connection service in that location where internet or cellular networks are impossible or at the situation of a disaster.

KEYWORDS: Cloud Computing; Mobile Ad Hoc Networks (MANET); Mobility Model; Software-Defined Networking (SDN). Received 29 Jan 2020 Revised 13 March 2020 Accepted 18 March 2020 Online 20 April 2020

(c) Transactions on Science and Technology

Review Article

\section{INTRODUCTION}

The wireless networking is one of the most progressive technology in the area of information technology for the last 50 years. In this era mostly organizations are using wireless networking to connect their offices. The MANET is one of the most useful networks that established dynamically among all connected devices without the fixed infrastructure in a decentralized approach (Alam \& Benaida, 2018a). Every smart device user is free to connect any other smart device (Alam \& Benaida, $2018 b)$. Every pair of smart devices has a way with various connections among them in the area of similar communication. It is expected that by 2020 , the development of the internet of smart devices connected exponentially with 50 billion smart devices. This development will not depend on mankind's population but to the reality that units utilized consistently. The reality of interconnectedness things is cooperating man to machines and machines to another machine. They will be talking with each other (Alam \& Aljohani, 2015a). The definition of the internet of things can be described as "a pervasive and ubiquitous system which empowers screening furthermore control of the physical earth by collecting, processing, also analyzing that information created eventually sensors" (Alam \& Aljohani, 2016; Alam \& Aljohani, 2015b). This evolutionary paradigm enables its users to deploy a connection to a network of computing resources in an effortless fashion, where users can rapidly scale up or down their demands with trivial interaction from service providers (Alam, 2017). Current and future networks are described in Figure 1. 

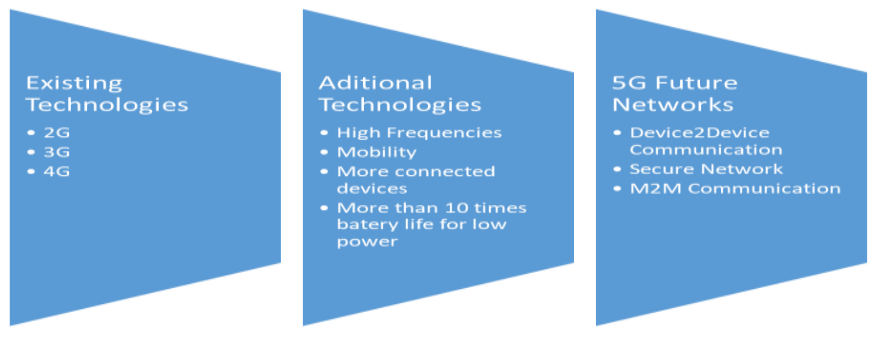

Figure 1. Current and Future Networks

\section{MOBILE AD HOC NETWORKS (MANET)}

MANET provides a connection at any area and no time-bound among smart devices if that area doesn't have the facility of the cellular network. Generally, it is used in military rescue operations or the disaster area (Alam, 2019a). MANET provides the best performance in that situation where we don't want centralized storage of the information for the example meeting room. Some private talk needs security or privacy, at that situation MANET helps us to create a virtual network without a centralized server, it is one of the various scenarios where MANETs perform excellently (Alam, 2018a). The MANET has a self-organizing decentralized approach that forms virtual communication (Alam, 2018b).

An ad hoc network of smart devices provides a facility to send messages from one smart device to another by using intermediate devices inside the entire network graph. For providing the best performance of smart devices network and avoiding the collisions problems using the proposed research is the main goal of our study. According to recent research on the message passing in the ad hoc network, there are using two techniques- one of them is deterministic and the other is the Probabilistic technique. The first technique is used to build a network backbone for covering all the devices in the entire network. Another technique is used to rebuild the backbone for every device and select the device probably and then send the message. The probability approach may very useful to find the best route for sending data among smart devices by using the fuzzy control system. The proposed research applied the fuzzy framework to find optimal Mobility Models in MANET of Smart Devices.

\section{SOFTWARE-DEFINED NETWORKING (SDN)}

SDN is a new interface that is real-time, optimized, effective, flexible, measurable and easy to adapt with high bandwidth. SDN is designed to separate the control plane and forwarding plane to enable the network control to become programmable that delivers decisions according to the specific network requirement (Mendonca et al., 2013). The software-defined networking has three layersApplication Layer, Control Layer, and forwarding layer. The SDN control plane works as the mastermind of networking (Xia et al., 2014). SDN is the intelligent technology that takes snapshots of the entire networks and their routing tables for instruct the forwarding plane to forward the network traffic to switches and routers. There are two kinds of APIs in SDN, the first is called South Bound API (SBAPI) and the second API is called North Bound API (NBAPI). It is an interface between the forwarding plane and the control plane. The SBAPI is used to insert information to the switches and routers and NBAPI inserts the information to the applications. It is an interface between network applications and control plane. 


\section{CLOUD SERVICES}

Security is a vast issue to be handled based on a single point of view. Various parties involved in the cloud paradigm have different objectives (Alam, 2019b). Therefore, they may vary their worries regarding threats and vulnerabilities in the cloud environment. Moreover, these worries might be eased or intensified depending on the implemented deployment model (Alam, 2020).

In the realism of the internet, security has been perceived as a prominent inhibitor of embracing the cloud paradigm. Since the cloud environment is a distributed architecture, which it's resource storage and management may lay in any place of the world, many concerns have been raised over its vulnerabilities, security threats and challenges. The involvement of various parties has widened these concerns based on each party's perspective and objective. It has been determined that the three dominant parties participate in the cloud environment (Alam, 2019c).

i. Service providers: Their concerns may be intensified over public and hybrid cloud where issues related to unauthorized access and cyber-attacks may jeopardize service availability.

ii. Service consumers: Their concerns may focus on issues related to data privacy and quality of service. Besides, their concerns regard service availability and interoperability.

iii. Service regulators: Their concerns may focus on issues related to service standard violations.

Thus, issues related to interoperability would affect them greatly. It is fair to say that all previously mentioned party concerns might be correlated and associated with other parties.

\section{MOBILITY MODELS}

Various pre-existing mobility models changes generally over the reality these start with totally simulated and extremely measurable practically. The review process of individuals' mobility modeling helps us to know the utilization of various mobility models in MANET (Alam \& Rababah, 2019). Figure 2 is describs the existing mobility models.

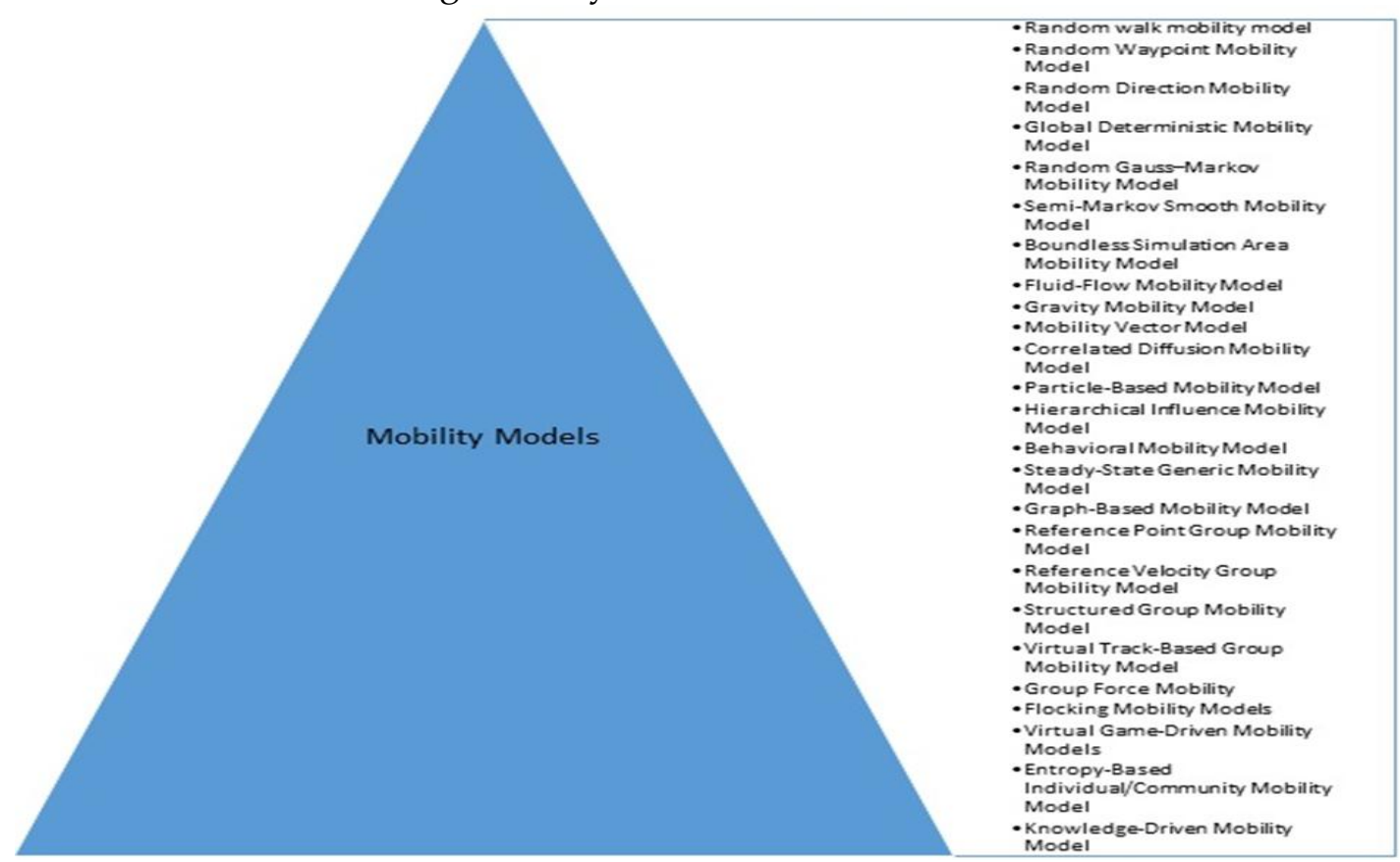

Figure 2. Classification of mobility models

The Ad Hoc means "just for this reason", it is a Latin expression for an autonomous collection of mobile nodes, with networks built on the fly for a specific purpose (i.e., emergencies, rescue operations, battlefield situations, etc.), that talk to each other over bandwidth-constrained wireless 
links. The proliferation of wireless portable devices as parts of everyday life, such as PDA, mobile phones, and laptops, etc. is progressing to add the facility of MANET inside the device for sharing the data among smart devices (Alam \& Benaida, 2020). However, communication over existing infrastructures may be precluded due to deficient facilities, or impractical in terms of time, expense and power. MANET contains an extraordinary subform that claiming a remote network without the server for transferring information from one smart device to another. The wireless network requires the basic fixed offices that are answerable to receive and sending the information among smart mobility devices, but the ad hoc network doesn't require this kind of fixed office.

\section{PROPOSED ARCHITECTURE}

The ad hoc network provides the facility to connect in a heterogeneous environment without a centralized approach. It is created automatically when two or more device has an active connection. The $4 \mathrm{G}$ network is implemented in almost cities over the world. 5G is the next-generation networking that is expected to implement by 2020. The implementation of a $5 \mathrm{G}$ network is no easy task because it will provide high-speed connectivity in real-time with very low latency. It also provides high security. The concept of Cloud Computing has been distinguished as one of the major computing models in recent years (Aljohani \& Alam, 2015). It came because of developments in previous computing paradigms which include parallel computing, grid computing, distributed computing, and other computing paradigms. The smart device's mobility causes incessant and flighty transforms the discretionary system topology for transforming the information. The Messages requiring a destination outside this local neighborhood zone must be hopped or transformed through the nearest smart device that is working as a router in the ad hoc network. The growth of the internet of things initially started in 2008 by connecting the physical objects to the internet. The physical objects relate to the smart database that has a collection of smart data. The framework has the image recognition technology for identifying the physical object, buildings, peoples, logo, location, etc. for business and customers. Now the internet of things is shifting from information-based technology to operational based technology i.e. IPV4 (man 2 machines) to IPV6 (machine 2 machines). It combines sensors, smart devices and interfaces like Smart Grid. The movements, positions, acceleration also velocity of smart device users are changed time by time. The purpose of MANET is to define the movements and analyze their purpose over time (Truong et al., 2015). So MANETs play a vital role to perform simulations of movements in the area of a wireless network. It can be very helpful for researchers in the area of mobility modeling. RWP mobility models are frequently used mobility model that is widely spreading useful in the area of Mobile Ad hoc Network. Presently researchers start focusing on the alternatives of the mobility models with advanced properties. Because of the mobility models more focus on the neighborhood's smart devices for creating the connections among smart devices so sometimes it takes time to spread information among the smart devices within the range of ad hoc networks. So many researchers proposed various types of mobility models for capturing different kinds of properties in real-time. In the Random walk mobility model, every smart device can move within the range of ad hoc network from one position to another position using the random directions as well as random velocity and speed. But the movement has been performed within the fixed timing and distance traveled. After that, the current directions will be selected. In the RWP mobility model, if the total number of smart devices within the area of the rectangle that should be the constant area. All smart devices can move randomly from one position to another position using randomly chosen way within the minimum also maximum velocity interval. When this device will arrive at its destinations then this device will choose the new speed and destination, after that this process will continue until it reaches the destination. 
In Random Direction Mobility Model, all smart devices users can be traveled in a simulation range with fixed speed and directions. After that the device stop then it discovers the latest directions also speed using the random selection. This process will repeat randomly. Figure 3 represents the Cloud-MANET mobility model for SDN. In Probabilistic Random Walk Mobility Model, the movements of smart device users using the probabilistic find directions from one location to another location. The Random Gauss-Markov Model is the improved model that allows the smart devices to move randomly using random walk mobility to the fluid flow. The Column Mobility Model is used to move the devices using a column line. It is very useful for discovering the device within the simulation area. Various researchers modify the column mobility model that allowed smart devices to discover neighborhood devices using a line. The smart devices are shifted to the reference location inside the refer grid after shifted the smart device then allow it for moving in a random refer location.

The Generalized Trace Based Mobility Model, the users of the smart device connected in MANET is an emerging technology that is allowed the access point for the connection creation process among the smart devices.

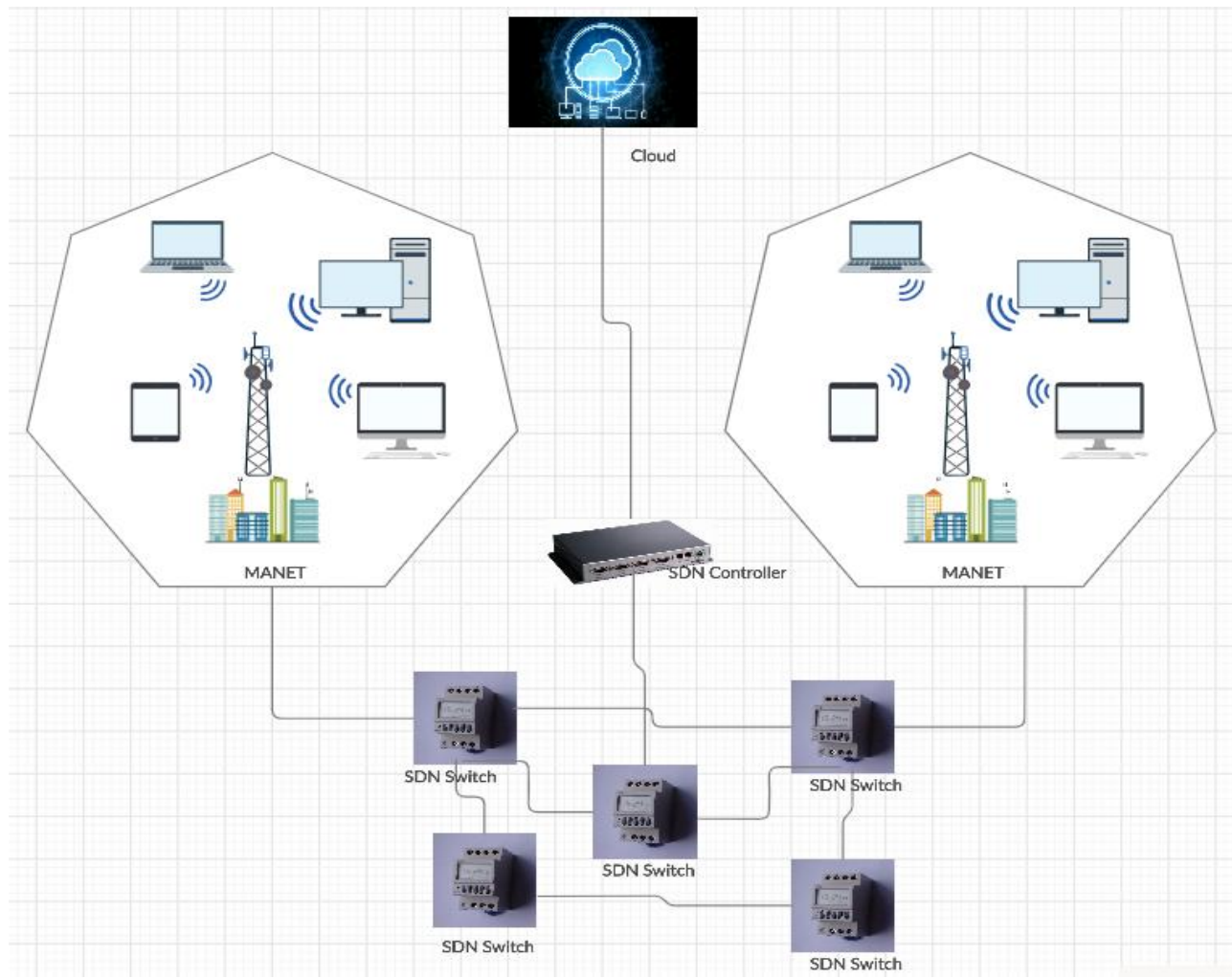

Figure 3. Cloud-MANET Mobility model for SDN

The smart device user's mobility in MANET determines the route of movement is the challenges and difficulties for predicting the movement of smart devices. The real tracing of smart users in a mobility environment is the real movement in the geographic simulated area. Sometimes these tracings perform accurately and result produced like real tracing. Various mobility models trace the movement of devices and collect the result for evaluating the performance. The boundless simulation area mobility model generates the torus-shaped simulated area that allows smart users to move from one location to another in a boundless simulation area. This coverage area will be folded in the $\mathrm{x}$-axis as well as the $\mathrm{y}$-axis and generate a cylinder. It can represent the closed coverage rectangular area mapping with the torus. The graph-based mobility model moves the smart device in the edge of the graphs in the network and visit all the vertices among the graph. The smart device users choose the vertex randomly in a graph and evaluate the simulation area of the ad hoc network. 


\section{CONCLUSION}

The MANETs are self-organizing decentralized networks without a fixed infrastructure of wireless smart gadgets that provide the facility to send and receive data among wireless smart devices within its transmission range. The information passing requires a destination outside this local neighborhood zone must be hopped or transferred through the nearest device to the appropriate target address. Because of node mobility fixed source/destination paths cannot be maintained for the lifetime of the network. The role of Software-defined networking in this model is to provide programmable networking and decoupling the control and data plane. It comes from the requirement for providing the dynamic, high speed with low latency connectivity in real-time. In this article, a new mobility model name as C-MANET Mobility Model (Cloud-based Mobile Ad Hoc Networks Mobility Model) in the architecture of software-defined networking (SDN) in 5G heterogeneous networks is found. The C-MANET mobility model provides the facility to connect to the internet of smart devices. The cloud services in MANET played a vital role at the time of disaster situations.

\section{REFERENCES}

[1] Alam, T. \& Aljohani, M. 2015a. An approach to secure communication in mobile ad-hoc networks of Android devices. Proocedings of the 2015 International Conference on Intelligent Informatics and Biomedical Sciences (ICIIBMS). 28-30 November 2015. Okinawa, Japan. pp. 371375.

[2] Alam, T. \& Aljohani, M. 2015b. Design and implementation of an Ad Hoc Network among Android smart devices. International Conference on Green Computing and Internet of Things (ICGCIoT). (pp. 1322-1327). IEEE. India.

[3] Alam, T. \& Aljohani, M. 2016. Design a new middleware for communication in ad hoc network of android smart devices. Second International Conference on Information and Communication Technology for Competitive Strategies. (pp. 1-6). India.

[4] Alam, T. \& Benaida, M. 2018a. The role of cloud-MANET framework in the internet of things (IoT). International Journal of Online Engineering, 14(12), 97-111.

[5] Alam, T. \& Benaida, M. 2018b. CICS: Cloud-Internet Communication Security Framework for the Internet of Smart Devices. International Journal of Interactive Mobile Technologies, 12(6), 74-84.

[6] Alam, T. \& Benaida, M. 2020. Blockchain, Fog and IoT Integrated Framework: Review, Architecture and Evaluation. Technology Reports of Kansai University. Vol 62 (02).

[7] Alam, T. \& Rababah, B. 2019. Convergence of manet in communication among smart devices in IoT. International Journal of Wireless and Microwave Technologies (IJWMT), 9(2), 1-10.

[8] Alam, T. 2017. Middleware Implementation in Cloud-MANET Mobility Model for Internet of Smart Devices. International Journal of Computer Science and Network Security, 17(5), 86-94.

[9] Alam, T. 2018a. A Reliable Communication Framework and Its Use in Internet of Things (IoT). International Journal of Scientific Research in Computer Science, Engineering and Information Technology (IJSRCSEIT), 3(5), 450-456.

[10] Alam, T. 2018b. A reliable framework for communication in internet of smart devices using IEEE 802.15. 4. ARPN Journal of Engineering and Applied Sciences, 13(10), 3378-3387.

[11] Alam, T. 2019a. 5G-Enabled Tactile Internet for smart cities: vision, recent developments, and challenges. Jurnal Informatika, 13(2), 1-10.

[12] Alam, T. 2019b. Blockchain and its Role in the Internet of Things (IoT). International Journal of Scientific Research in Computer Science, Engineering and Information Technology, 5(1), 151-157.

[13] Alam, T. 2019c. IoT-Fog: A communication framework using blockchain in the internet of things. International Journal of Recent Technology and Engineering (IJRTE), 7(6), 833 - 838. 
[14] Alam, T. 2020. Internet of Things: A Secure Cloud-Based MANET Mobility Model. International Journal of Network Security, 22(3), 514 - 520.

[15] Aljohani, M. \& Alam, T. 2015. An algorithm for accessing traffic database using wireless technologies. IEEE International Conference on Computational Intelligence and Computing Research (ICCIC). (pp. 1-4). IEEE. India.

[16] Mendonca, M., Astuto, B.N., Obraczka, K. \& Turletti, T. 2013. Software defined networking for heterogeneous networks. IEEE MMTC E-Letters, IEEE, 8 (3), 36-39.

[17] Truong, N.B., Lee, G.M. \& Ghamri-Doudane, Y. 2015. May. Software defined networking-based vehicular adhoc network with fog computing. IFIP/IEEE International Symposium on Integrated Network Management (IM), (pp. 1202-1207). IEEE. Canada.

[18] Xia, W., Wen, Y., Foh, C.H., Niyato, D. \& Xie, H. 2014. A survey on software-defined networking. IEEE Communications Surveys \& Tutorials, 17(1), 27-51. 